\title{
Factores de riesgo para mortalidad en la infección por Pseudomonas aeruginosa en pacientes oncológicos hospitalizados en tres ciudades de Colombia
}

\author{
Risk Factors for Mortality Caused by Pseudomonas Aeruginosa \\ Infection in Hospitalized Patients with Oncologic Diagnosis in \\ three Cities of Colombia
}

\section{Fatores de risco de mortalidade na infecção por Pseudomonas aeruginosa em pacientes com câncer hospitalizado em três cidades na Colômbia}

\author{
David Cataño-Toro, MD., Esp. * \\ José William Martinez, MD., MSc. ** \\ Manuel A. Martínez-Muñoz., Est.*** \\ Juliet J. López-Osorio., Est.***
}

\author{
Daniel S. Marín-Medina., Est. *** \\ Juan P. Orozco-Hernández., Est.*** \\ Juan D. Sosa-Urrea., Est.*** \\ Jorge A. Sánchez-Duque., Est.***
}

\begin{abstract}
Resumen
Introducción: Pseudomonas aeruginosa es una bacteria oportunista Gram negativa particularmente eficiente en la adquisición de mecanismos de resistencia y de alta prevalencia en infecciones nosocomiales en pacientes oncológicos. Objetivo: identificar los factores de riesgo para mortalidad en pacientes oncológicos con aislamiento de $P$. aeruginosa. Metodología: estudio descriptivo, la población de estudio fueron los casos reportados con aislamiento de $P$. aeruginosa en el servicio de hospitalización de Oncólogos de Occidente en Pereira, Armenia y Manizales durante el año
\end{abstract}

2015. Se realizaron análisis univariados y multivariados; la supervivencia se estableció según el método de KaplanMeier. Se estableció un valor de $p<0.05$. Se usó el software STATA. Se tuvo aval de bioética de la Universidad Tecnológica de Pereira. Resultados: se estudió 41 casos confirmados de cultivos positivos de $P$. aeruginosa. El sexo masculino (46.3\%), anemia (46.3\%), neutropenia febril (41\%), trombocitopenia (29.3\%) y haber sido hospitalizado en la unidad de cuidados intensivos (29.3\%) fueron asociados estadísticamente con mayor mortalidad $(p=0.019)$; con estos resultados se diseñó una escala de riesgo (alfa de Cronbach $=0.72$ ). Los pacientes con cuatro de

* Médico, Especialista en medicina interna, Universidad Tecnológica de Pereira, Pereira, Risaralda, Colombia.

** Médico, Especialista en epidemiología, Docente titular Universidad Tecnológica de Pereira, Pereira, Risaralda, Colombia.

*** Estudiante de medicina, Universidad Tecnológica de Pereira, Grupo de Investigación epidemiología, Salud y violencia, Facultad de Ciencias de la Salud, Universidad Tecnológica de Pereira, Pereira, Risaralda, Colombia.

Correspondencia: Jorge Andrés Sánchez Duque, Carrera 27 No. 10-02 Barrio Álamos, Universidad Tecnológica de Pereira, Edificio 14, Facultad de Ciencias de la Salud, Piso 3, Ciencias comunitarias. Teléfono: + 57 3137032170. Email: jorandsanchez@utp.edu.co 
estas exposiciones mostraron mayor riesgo de mortalidad a egreso hospitalario con una sensibilidad del 68\% y especificidad del $90 \%$. La $P$. aeruginosa presentó resistencia a cefepime $(36.6 \%)$ y a aztreonam $(34.1 \%)$, mientras que la letalidad global fue del $26.8 \%$. Conclusión: El sexo masculino, la coexistencia de anemia, trombocitopenia, y neutropenia febril, así como la estancia en la unidad de cuidados intensivos aumentan la mortalidad en los pacientes oncológicos infectados con $P$. aeruginosa. [Cataño-Toro $D$, Martínez JW, Martínez-Muñoz MA, López-Osorio JJ, MarínMedina DS, Orozco-Hernández JP, Sosa-Urrea JD, Sánchez-Duque JA. Factores de riesgo para mortalidad en la infección por Pseudomonas aeruginosa en pacientes oncológicos hospitalizados en tres ciudades de Colombia. MedUNAB 2017; 20(1): 39-47].

Palabras clave: Pseudomonas aeruginosa; Neoplasias; Infección Hospitalaria; Farmacorresistencia Microbiana; Colombia.

\section{Abstract}

Introduction: Pseudomonas aeruginosa is a Gram-negative and rod-shape opportunistic bacterium that is particularly efficient in the acquisition of resistance mechanisms and its high prevalence in nosocomial infections in cancer patients. Objective: To identify risk factors for mortality in cancer patients with $P$. aeruginosa infection. Methodology: A descriptive study was carried out in patients with $P$. aeruginosa infection during the hospitalization service of "Oncólogos de Occidente" in Pereira, Armenia and Manizales during 2015. Univariate and multivariate analyzes were performed. The survival analysis was established according to the Kaplan-Meier method. A value of $p<0.05$ was established for it. The analyses were examined with the STATA software. This study was endorsed by the bioethics committee of the "Universidad Tecnológica de Pereira". Results: Fourty-one patients with positive culture for $P$. aeruginosa were studied. Males (46.3\%), anemia (46.3\%), febrile neutropenia (41\%), thrombocytopenia (29.3\%) and previous hospitalization in an intensive care unit $(29.3 \%)$ were associated with higher mortality risk ( $p=0.019)$; a risk scale was designed with these factors (Cronbach's alpha $=0.72$ ). Patients who presented four of these exposures were at higher risk of mortality with a sensitivity of $68 \%$ and specificity of $90 \%$ at the moment of discharge. $P$. aeruginosa showed $36.6 \%$ of resistance to cefepime, $34.1 \%$ to aztreonam, the mortality rate was $26.8 \%$. Conclusion: Male sex, anemia, thrombocytopenia, febrile neutropenia and previous hospitalization in an intensive care unit increase the mortality rate in patients with cancer who were infected by $P$. aeruginosa. [Cataño-Toro D, Martínez JW, Martínez-Muñoz

\section{Introducción}

Las terapias antineoplásicas actuales han incrementado la expectativa de vida de los pacientes oncológicos pero han aumentado el riesgo de infecciones asociadas a inmunosupresión. Anteriormente, las bacterias Gram positivas eran las causantes de la mayoría de infecciones en pacientes oncológicos, tendencia que ha ido cambiando en la
MA, López-Osorio JJ, Marín-Medina DS, Orozco-Hernández JP, Sosa-Urrea JD, Sánchez-Duque JA. Risk Factors for Mortality Caused by Pseudomonas Aeruginosa Infection in Hospitalized Patients with Oncologic Diagnosis in three Cities of Colombia. MedUNAB 2017; 20(1): 39-47].

Keywords: Pseudomonas aeruginosa; Neoplasms; Cross Infection; Drug Resistance, Microbial; Colombia

\section{Resumo}

Introdução: Pseudomonas aeruginosa é uma bactéria oportunista Gram-negativa particularmente eficiente na aquisição de mecanismos de resistência e alta prevalência em infecções nosocomiais em pacientes com câncer. Objetivo: identificar os fatores de risco para mortalidade em pacientes oncológicos com isolamento de P. aeruginosa. Materiais e métodos: estudo descritivo, a população estudada foram os casos relatados com isolamento de $\mathrm{P}$. aeruginosa ao serviço da hospitalização de Oncologistas do Ocidente em Pereira, Armênia e Manizales durante o ano de 2015. Foram realizadas análises univariadas e multivariadas; a sobrevivência foi estabelecida de acordo com o método de Kaplan-Meier. Foi estabelecido um valor de p. <0,05 y se utilizou o software STATA, aprovada pelo Comité de Bioética da Universidade Tecnológica de Pereira. Resultados: 41 casos confirmados de P. aeruginosa culturas positivas foram estudadas. A anemia (46,3\%), a neutropenia febril (41\%), a trombocitopenia $(29,3 \%)$ e a hospitalização na unidade de terapia intensiva (29,3\%) foram associadas estatisticamente com maior mortalidade (p. = 0,019); Com esses resultados, foi elaborada uma escala de risco (Alfa de Cronbach $=0,72$ ). Os pacientes com quatro dessas exposições apresentaram maior risco de mortalidade na alta hospitalar com uma sensibilidade de $68 \%$ e uma especificidade de $90 \%$. P. aeruginosa apresentou resistência ao cefepima $(36,6 \%)$ e aztreonam $(34,1 \%)$, enquanto a letalidade global foi de $26,8 \%$. Conclusão: o sexo masculino, a coexistência de anemia, trombocitopenia e neutropenia febril, além de permanecer na unidade de terapia intensiva, aumentam a mortalidade em pacientes oncológicos infectados com $P$. aeruginosa. [Cataño-Toro D, Martínez JW, Martínez-Muñoz MA, LópezOsorio JJ, Marín-Medina DS, Orozco-Hernández JP, SosaUrrea JD, Sánchez-Duque JA. Fatores de risco de mortalidade na infecção por Pseudomonas aeruginosa em pacientes com câncer hospitalizado em três cidades na Colômbia. MedUNAB 2017; 20(1): 39-47].

Palavras-chave: Pseudomonas aeruginosa; Neoplasias; Infecção Hospitalar; Resistência Microbiana a Medicamentos; Colômbia.

última década, en la cual las bacterias Gram negativas como $P$. aeruginosa y E. coli gobiernan este panorama $(1,2)$. El aumento de infecciones por Gram negativos es multifactorial en los pacientes oncológicos dado que en ellos, además de presentarse una respuesta inmunitaria disminuida, hay una mayor exposición a equipos invasivos como el uso continuo de catéteres venosos centrales propios de la quimioterapia e inclusive el consumo profiláctico de fluoroquinolonas (3). 
Las infecciones del torrente sanguíneo son las más frecuentes y severas en estos pacientes alcanzando una mortalidad superior al $40 \%(2)$.

La tasa de infecciones intrahospitalarias van del 3.8 al $18.6 \%$, pero en pacientes oncológicos se reportan prevalencias aún mayores $(4,5)$. La resistencia a antimicrobianos es uno de los mayores problemas de salud pública a nivel mundial, por lo cual el monitoreo de patrones de susceptibilidad a los antimicrobianos es determinante para la evaluación de los regímenes de terapia empírica y no empírica permitiendo generar alertas a los profesionales involucrados en la atención sobre la presencia de mecanismos de resistencia inusuales o emergentes(6-10). El origen de este problema radica en el uso inapropiado de antibióticos, la falta de sistemas de vigilancia efectivos, la ausencia de legislación que permita el control en el mercado y el uso extendido de antibióticos en animales (11). En los peores casos aparece la resistencia a tres o más clases de antibióticos activos contra un germen, haciéndolo multirresistente (9). Ante este desalentador futuro no son muchas las alternativas farmacológicas disponibles $(12,13)$.

$P$. aeruginosa es una bacteria oportunista Gram negativa que crece en una amplia variedad de medios aerobios y anaerobios, la cual ha sido aislada en superficies húmedas de los pacientes como las axilas, el oído, periné y tejidos macerados, así como en los entornos húmedos de las duchas, lavados, sanitarios, entre otros (14-17). P. aeruginosa es particularmente eficiente en la adquisición de mecanismos de resistencia que están involucradas en la génesis de infecciones nosocomiales, lo cual hace más difícil la terapia antibiótica empírica y guiada por antibiograma aumentando la mortalidad, estancia hospitalaria y costos asociados al cuidado del paciente oncológico $(8,10,18)$. La presencia de infección por $P$. aeruginosa ha sido reportada anteriormente en el $10 \%$ de los pacientes con patologías oncológicas hospitalizados(19). Se ha aislado $P$. aeruginosa en pacientes con neoplasias hematológicas en un $47.6 \%$ del total de los aislamientos en estos pacientes (20), y aproximadamente en el $17 \%$ de los asilamientos en pacientes con cáncer(21). La incidencia de bacteriemia por P. aeruginosa ha disminuido ( 2.8 vs 4.7 casos por cada 1000 hospitalizaciones) $(22,23)$. Se ha descrito una frecuencia del $6 \%$ de infección intrahospitalaria por $P$. aeruginosa en el Instituto nacional de cancerología de Colombia(24).

En este estudio se describen las variables que impactan la mortalidad de pacientes oncológicos con aislamientos de $P$. aeruginosa durante su hospitalización en Oncólogos del Occidente en Armenia, Manizales y Pereira.

\section{Metodología}

Se realizó un estudio descriptivo de corte transversal para evaluar los factores de riesgo que condicionan la mortalidad en los pacientes oncológicos que adquirieron una infección por $P$. aeruginosa. Los criterios de inclusión fueron: 1) mayor de 18 años, 2) con diagnóstico oncológico, 3) haber sido hospitalizado en Oncólogos de occidente en Armenia, Manizales o Pereira durante el año 2015 y 4) cultivo positivo para $P$. aeruginosa durante la hospitalización. No se excluyó a ningún paciente.

Variables: Se recolectó información sociodemográfica, diagnóstico oncológico, cuadro hemático completo, presencia de neutropenia febril (se define por la presencia de un conteo absoluto de neutrófilos $<500 / \mathrm{mL}$ y la presencia de fiebre, definida según las guías NCCN (National Comprehensive Cancer Network) e IDSA (Infectious Disease Society of America) como temperatura oral $>38.5^{\circ} \mathrm{C}$ en un solo momento en ausencia de causas ambientales obvias o $>38.3^{\circ} \mathrm{C}$ por al menos una hora $(25$, 26); Se calculó índice de Charlson y puntaje de MASCC (Escala de riesgo de MASCC por Multinational Association for Supportive Care in Cancer), tiempo de estancia hospitalaria e ingreso UCI (Unidad de cuidado intensivo), características microbiológicas de los cultivos de $P$. aeruginosa y supervivencia de los pacientes.

Fuente de información: todos los pacientes quienes durante su estancia hospitalaria en Oncólogos se encuentren febriles o tengan un recuento de neutrófilos por debajo de 1,500 son sistemáticamente cultivados para establecer un foco infeccioso. El laboratorio clínico reporta a través de WHONET 5.6 (Aplicación diseñada para la recolección y análisis de información proveniente de los laboratorios de microbiología) la fecha de toma de la muestra, la fuente de la muestra que resulta positiva, el agente, la concentración inhibitoria mínima, formación de resistencia antimicrobiana in vitro y define la resistencia bacteriana según las guías CLSI 2015 (The Clinical and Laboratory Standards Institute), que además son incorporadas por WHONET. La información demográfica y clínica de los pacientes se extrajo del sistema de historias clínicas oncológicas SAHICO. Posteriormente se analizaron los resultados del antibiograma con el sistema Epicenter establecidos por las guías CLSI 2015. En el caso de un reporte poco común se confirmó el resultado reiniciando el proceso desde la siembra.

Procesamiento de la información: con la identificación de pacientes se mezclaron las bases de datos de SAHICO y WHONET. El Comité de Infecciones de Oncólogos del Occidente estudia los casos y discute la información para orientar las intervenciones de control normadas para el control de brotes y consideró una $P$. aeruginosa resistente a múltiples fármacos si muestra resistencia (intermedia o completa) al menos a tres de los siguientes cuatro grupos de antibióticos: carbapenémicos (imipenem o meropenem); cefalosporina de tercera o cuarta generación (ceftazidima o cefepima); productora de betalactámicos (piperacilina o piperacilina / tazobactam); y quinolonas (ciprofloxacina o levofloxacina). 
Análisis de datos: Se analizaron las medianas y rango intercuartílico (RIQ) de las variables continuas y discretas. Las variables nominales se analizaron a través de frecuencias absolutas y relativas. Para el análisis de supervivencia se utilizó el método de Kaplan-Meier. Se estableció un modelo multivariado que generó el mejor modelo para la explicación de la muerte de los pacientes con infección por $P$. aeruginosa. Se estableció significancia estadística con un valor de $\mathrm{p}<0.05$. Todos los análisis se hicieron con el software STATA versión 14.2 versión oficial.

Los grupos de bioética son convocados para proteger los derechos, guardar el bienestar de los participantes y determinar los riesgos de su inclusión; por ello el trabajo fue aprobado por parte del comité de bioética de la universidad tecnológica de Pereira en la categoría de "investigación sin riesgo", según la resolución No. 8430 de 1993 del Ministerio de Salud de Colombia, que establece las normas científicas, técnicas y administrativas para la investigación en salud.

\section{Resultados}

Para el año de estudio, la institución reportó 1,814 egresos que correspondieron a 968 pacientes, estos generaron en total 21,364 días de estancia hospitalaria. 41 pacientes fueron identificados como infectados por $P$. aeruginosa por cultivo, para una tasa de infección de 2 pacientes por cada 1,000 días de estancia hospitalaria. La prevalencia establecida fue de 2 pacientes con $P$. aeruginosa por cada 100 egresos. $22(53.7 \%)$ de los 41 pacientes fueron mujeres y el $43.9 \%$ son de 50 o más años de edad. El 73.2\% pertenecían al régimen contributivo y el $46 \%$ permanecen casados o en unión libre.

En la Tabla 1 se observa que los diagnósticos oncológicos más prevalentes fueron linfoma no Hodgkin, síndrome mielodisplásico y leucemia mieloide aguda con un $19.5 \%$, $12.1 \%$ y $9.7 \%$ respectivamente. El $92.7 \%$ de los pacientes ingresó con anemia, el $43 \%$ cursó con leucopenia y el 29.3\% se reportó con cifras bajas de plaquetas (Tabla 2).

Tabla 1. Diagnóstico oncológico inicial en los pacientes con aislamiento de P. aeruginosa.

\begin{tabular}{lc}
\hline Diagnóstico & $\mathbf{N}(\%)$ \\
\hline Linfoma no Hodgkin & $9(21.9)$ \\
\hline Síndrome Mielodisplásico & $6(14.6)$ \\
\hline Leucemia linfoblástica aguda & $3(7.3)$ \\
\hline Mieloma múltiple & $3(7.3)$ \\
\hline Tumor maligno de piel & $2(4.9)$ \\
\hline Otras patologías oncológicas * & $18(43.9)$ \\
\hline
\end{tabular}

* Tumor maligno de piel, tumor maligno del recto, tumor maligno del retro peritoneo, tumor maligno del riñón, tumor maligno del fundus gástrico, tumor maligno del conducto anal, tumor maligno de colon, tumor maligno de páncreas, tumor maligno de vejiga, tumor maligno de cabeza y cuello, tumor de comportamiento incierto, histiocitosis de las células de Langerhans, linfoma de Hodgkin.

Tabla 2. Hemoleucograma en pacientes con aislamiento de P. aeruginosa.

\begin{tabular}{lrr}
\hline Variable & Media & IC 95\% \\
\hline Hemoglobina inicial (g/dl) & 9.9 & $9.19-10.5$ \\
\hline Hematocrito inicial (\%) & 29.8 & $27.8-31.8$ \\
\hline Recuento inicial de leucocitos & 6886.0 & $5,074.9-8,697$ \\
\hline Plaquetas & $246,967.3$ & $193,828-300,106.6$ \\
\hline Recuento inicial de neutrófilos & $4,703.6$ & $3,069.2-3,069.2$ \\
\hline
\end{tabular}


La Tabla 3 muestra que el $46 \%$ de los pacientes reportaron leucopenias y el $41 \%$ de los pacientes fueron neutropénicos febriles (fiebre y recuento de neutrófilos inferior a 500 por cc) $(20,21)$. Estos pacientes fueron sistemáticamente cultivados para bacterias y hongos. La mediana de edad de los no neutropénicos febriles fue de 53 años mientras que los neutropénicos febriles reportaron una mediana de edad de 14 años.

Los pacientes infectados con $P$. aeruginosa presentan una muy alta frecuencia de anormalidades hematológicas y el antecedente de haber sido hospitalizados en la unidad de cuidados intensivos es bastante frecuente. Después del análisis multivariado, aquellas variables con asociación significativa fueron procesadas como una escala, incluyendo las siguientes variables categóricas: neutropenia febril, anemia, trombocitopenia, estancia en UCI y sexo masculino. Cada variable fue considerada como una exposición y se relacionaron con el desenlace de egreso de los pacientes, con lo que se encontró una asociación significativa con un valor de $\mathrm{p}=0.019$ estimado por un test exacto de Fisher (Tabla 4). La letalidad en los pacientes con $P$. aeruginosa fue del $26.8 \%$.

Tabla 3. Condiciones clínicas de los pacientes neutropénicos febriles, $n=17$

\begin{tabular}{lrr}
\hline Variable & Media & IC95\% \\
\hline Índice de Charlson* & 4.0 & $3.2-5.0$ \\
\hline Escala MASCC** & 17.1 & $15.4-18.8$ \\
\hline Hemoglobina (g/dl) & 9.0 & $8.4-9.6$ \\
\hline Hematocrito (\%) & 26.5 & $24.6-28.5$ \\
\hline Leucocitos & 822.1 & $429.2-1,215$ \\
\hline Neutrófilos & 225.8 & $101.9-349.7$ \\
\hline Plaquetas & $6,8842.1$ & $46,352.8-91,331.4$ \\
\hline Temperatura ${ }^{\circ} \mathrm{C}$ & 37.7 & $37.2-38.1$ \\
\hline Número de picos febriles & 4.3 & $2.8-5.9$ \\
\hline Duración de la fiebre (días) & 16.1 & $5.9-26.3$ \\
\hline Días estancia en la UCl*** & 9.2 & $3.5-14.9$ \\
\hline Estancia hospitalaria (días) & 30.8 & $24.1-37.6$ \\
\hline
\end{tabular}

* Índice de comorbilidades de Charlson: relaciona la mortalidad a largo plazo con la comorbilidad del paciente (22).

** Escala de MASCC (Escala de riesgo de MASCC por Multinational Association for Supportive Care in Cancer) (23).

*** $\mathrm{UCl}$ (Unidad de cuidados intensivos).

Tabla 4. Exposiciones de riesgo y estado de los pacientes al egreso

\begin{tabular}{lcc}
\hline Suma de exposiciones de riesgo & Muerto & Vivo \\
\hline $\mathbf{1}$ & 0 & 2 \\
\hline $\mathbf{2}$ & 3 & 9 \\
\hline $\mathbf{3}$ & 3 & 16 \\
\hline $\mathbf{4}$ & 1 & 3 \\
\hline $\mathbf{5}$ & 4 & 0 \\
\hline Total & 11 & 30 \\
\hline
\end{tabular}


Se realizó un análisis de componentes principales con el que se estableció dos dimensiones, pero el ser hombres no discriminó las dos. A la lista de exposiciones de riesgo para los pacientes infectados por $P$. aeruginosa se le calculó un alfa de Cronbach que se reportó en 0.7 y se obtuvo las cargas factoriales para cada dimensión (Tabla 5).

En la Figura 1 se observa que si se obtiene cuatro puntos de la lista de exposiciones de riesgo se alcanza una sensibilidad de $68 \%$ para la clasificación del riesgo de muerte, con una especificidad del $90 \%$.
La $P$. aeruginosa presenta sensibilidades mayores al $70 \%$ para amikacina, ciprofloxacina, colistina, gentamicina y meropenem. Se encontró sensibilidad intermedia y resistencia del $30 \%$ o más para aztreonam, cefepime, imipenem y piperacilina tazobactam como se muestra en el Tabla 6.

Para el análisis del perfil microbiológico de $P$. aeruginosa se tuvieron en cuenta 22 hemocultivos, 10 urocultivos, 6 muestras de foco cutáneo y 3 muestras extraídas por lavado bronquial. No se aisló ninguna $P$. aeruginosa multirresistente.

Tabla 5. Exposiciones de riesgo y cargas factoriales.

\begin{tabular}{llll}
\hline Variable & Frecuencia (\%) & Factor 1 & Factor 2 \\
\hline Neutropenia febril & $17(41.5)$ & 0.929 & \\
\hline Trombocitopenia & $12(29.3)$ & & 0.892 \\
\hline Anemia & $19(46.3)$ & 0.939 & \\
\hline Estancia en UCl & $12(29.3)$ & -0.766 & \\
\hline Sexo masculino & $19(46.3)$ & & 0.563 \\
\hline
\end{tabular}

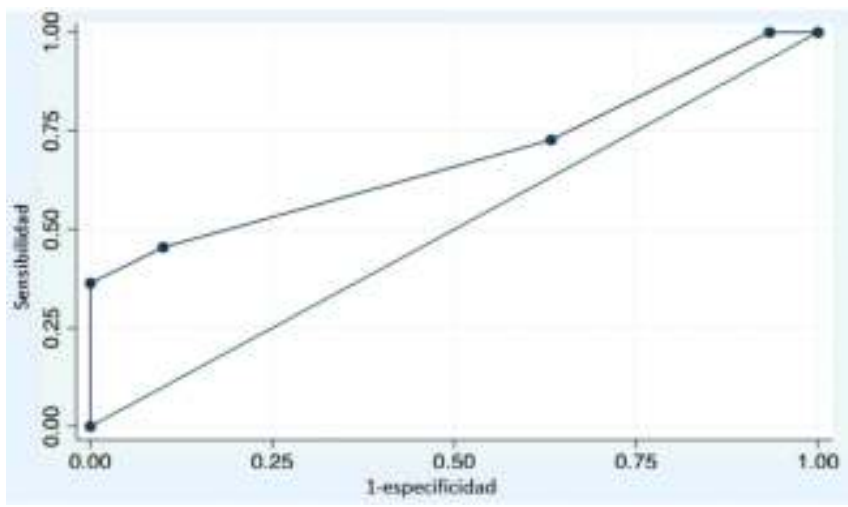

Figura 1. Sensibilidad y especificidad de cada punto de corte frente al riesgo de un desenlace fatal.

Tabla 6. Perfil microbiológico de la $P$. aeruginosa en población oncológica.

\begin{tabular}{lcc}
\hline Antibiótico & Sensible (\%) & Intermedio/resistente (\%) \\
\hline Amikacina & 73.2 & 26.8 \\
\hline Aztreonam & 65.9 & 34.1 \\
\hline Cefepime & 63.4 & 36.6 \\
\hline Ciprofloxacina & 75.6 & 24.4 \\
\hline Colistina & 92.7 & 7.3 \\
\hline Gentamicina & 82.9 & 17.1 \\
\hline Imipenem & 70.7 & 29.3 \\
\hline Meropenem & 78.1 & 21.9 \\
\hline Piperacilina/tazobactam & 70.7 & 29.3 \\
\hline
\end{tabular}




\section{Discusión}

En este estudio el $41 \%$ de los pacientes cumplió criterios para neutropenia febril durante la hospitalización en la que tuvo aislamiento de $P$. aeruginosa. La neutropenia febril es una complicación grave del paciente en manejo de patologías oncológicas (25), la cual se asocia a la toxicidad directa de los tratamientos antineoplásicos y en quienes se presentan tasas de mortalidad intrahospitalaria del 7-11\%, además de que conlleva retrasos en la continuación de la quimioterapia y, en muchos casos, reducción de dosis de los subsiguientes ciclos (26). Las tasas de mortalidad por esta complicación varían de acuerdo al diagnóstico, siendo del $14.3 \%$ para leucemias agudas y síndromes mielodisplásicos, $8.9 \%$ para linfomas y $8 \%$ para tumores sólidos (27), que son los principales diagnósticos en la población de este estudio. En estos pacientes la mayoría tuvo un índice MASCC menor a 21 que predice un mayor riesgo de presentar complicaciones derivadas de la neutropenia febril: entrada a UCI, falla respiratoria, falla renal, sangrado entre otras (28, 29). Además, la mayoría de pacientes con neutropenia febril presentó al menos otras dos comorbilidades evaluadas por el índice de Charlson que incluyen: diabetes, SIDA, patología renal, EPOC, infarto de miocardio, insuficiencia cardíaca, enfermedad cerebrovascular entre otras que indican una disminución en la supervivencia (29), y que deben ser tenidas en cuenta de manera individual.

Casi el 30\% de los pacientes presentó trombocitopenia, la cual ha sido identificada como un marcador temprano de progresión hacia la falla orgánica múltiple en pacientes con infecciones (30). La trombocitopenia y la anemia son frecuentes en las infecciones por $P$. aeruginosa ya que produce endotoxinas causantes de supresión en la médula ósea (31). En pacientes con bacteriemia por $P$. aeruginosa se ha visto que la trombocitopenia está asociada con una mayor mortalidad a los 30 días(32).

El 29.3\% de los pacientes estuvo en la UCI lo cual indica que tienen otros factores agravantes que podrían aumentar la mortalidad y que no fueron posibles de captar en este estudio. Además, un importante porcentaje de los pacientes infectados por $P$. aeruginosa tenía neoplasias hematológicas que establecen un riesgo de mortalidad específico según el grupo de edad en que se presentan, los esquemas antineoplásicos empleados, la progresión clínica entre otros que deben ser evaluados en cada paciente.

En nuestro estudio, la resistencia de $P$. aeruginosa aislada en pacientes oncológicos a amikacina ronda el $26 \%$, otros estudios reportan resistencias similares $26 \%$ (33), $32 \%$ (34), $50 \%$ (35); la resistencia ante aztreonam fue de $34 \%$, inferior al $45 \%$ reportado por el Dr. Zafer (34); la resistencia a cefepime fue de $36 \%$, inferior a lo reportado por el Dr. Trecarichi (35); la resistencia a ciprofloxacina fue de $24 \%$ frente a $25 \%(33), 43 \%(34), 63 \%(35)$; la resistencia a gentamicina fue de $17 \%$, frente a $50 \%$ reportado por el Dr. Zafer (34). La resistencia a imipenem fue de $29 \%$, otros estudios reportan $26 \%$ (33), 39\% (34) y $60 \%$ (35); el meropenem reportó resistencia en $21 \%$, inferior a otros estudios $46 \%$ (34) y $60 \%$ (35); la resistencia a la piperacilina/tazobactam fue de $29 \%$, frente a $24 \%$ (35), $25 \%$ (34) y $43 \%(33)$.

Es de resaltar que este estudio logró encontrar factores de riesgo fácilmente evaluables por medio del hemograma, el cual se obtiene de manera periódica, con lo que se puede identificar de manera precoz la evolución clínica de los pacientes. Las otras variables de exposición son también de fácil recolección (estancia en UCI y sexo masculino) por lo que se dispone de una escala sencilla que tiene una consistencia interna aceptable (alfa de Cronbach 0.72) que permitirá tener un apoyo objetivo en la valoración individual de los pacientes oncológicos en quienes se demuestra una infección por $P$. aeruginosa.

A pesar de haberse incluido tres instituciones de referencia en oncología en todo el eje cafetero, el tamaño muestral sigue siendo pequeño por lo que la validez externa de los hallazgos de este estudio no es la mejor. Sin embargo, estudios más grandes podrán establecer factores de riesgo en el diverso grupo de infecciones que presentan los pacientes oncológicos y que puedan ser intervenidas tempranamente.

\section{Conclusión}

La infección por Pseudomonas aeruginosa en los pacientes con patología oncológicas tiene una alta letalidad y resistencia antibiótica, razón por la cual es importante sospecharla durante la hospitalización de estos pacientes, caracterizar sus rasgos microbiológicos y dirigir un tratamiento de acuerdo a esto. Se debe tener en cuenta que el sexo masculino y la coexistencia de anemia, trombocitopenia, neutropenia febril y estancia en la unidad de cuidados intensivos aumentan la mortalidad, por lo que una identificación temprana ayudará a mejorar el pronóstico en estos pacientes.

\section{Conflicto de intereses}

Los autores manifiestan que no tienen ningún conflicto de interés.

\section{Agradecimientos}

Al personal del laboratorio clínico de Oncólogos de Occidente en Pereira (Colombia). 


\section{Referencias}

1. Bodey GP. Pseudomonas aeruginosa infections in cancer patients: have they gone away? Curr Opin Infect Dis. 2001;14(4):403-7.

2. Hidron AI, Edwards JR, Patel J, Horan TC, Sievert DM, Pollock DA, et al. NHSN annual update: antimicrobialresistant pathogens associated with healthcareassociated infections: annual summary of data reported to the National Healthcare Safety Network at the Centers for Disease Control and Prevention, 2006-2007. Infect Control Hosp Epidemiol. 2008;29(11):996-1011.

3. Bou R, Aguilar A, Perpinan J, Ramos P, Peris M, Lorente $L$, et al. Nosocomial outbreak of Pseudomonas aeruginosa infections related to a flexible bronchoscope. J Hosp Infect. 2006;64(2):129-35.

4. Molina F, Díaz C, Barrera L, De La Rosa G, Dennis R, Dueñas $\mathrm{C}$, et al. Perfil microbiológico de la Infecciones en Unidades de Cuidados Intensivos de Colombia (EPISEPSIS Colombia). Medicina Intensiva. 2011;35(2):75-83.

5. Jroundi I, Khoudri I, Azzouzi A, Zeggwagh AA, Benbrahim NF, Hassouni F, et al. Prevalence of hospitalacquired infection in a Moroccan university hospital. American journal of infection control. 2007;35(6):412-6.

6. Dobbs TE, Guh AY, Oakes P, Vince MJ, Forbi JC, Jensen B, et al. Outbreak of Pseudomonas aeruginosa and Klebsiella pneumoniae bloodstream infections at an outpatient chemotherapy center. Am J Infect Control. 2014;42(7):731-4.

7. Falagas ME, Kopterides P. Risk factors for the isolation of multi-drug-resistant Acinetobacter baumannii and Pseudomonas aeruginosa: a systematic review of the literature. Journal of Hospital Infection. 2006;64(1):7-15.

8. Giamarellou H. Multidrug-resistant Gram-negative bacteria: how to treat and for how long. International Journal of Antimicrobial Agents. 2010;36, Supplement 2(0):S50-S4.

9. Briceno DF, Correa A, Valencia C, Torres JA, Pacheco R, Montealegre MC, et al. Antimicrobial resistance of Gram negative bacilli isolated from tertiary-care hospitals in Colombia. Biomedica : revista del Instituto Nacional de Salud. 2010;30(3):371-81.

10. Martínez Buitrago E, Hernández C, Pallares C, Pacheco $\mathrm{R}$, Hurtado K, Recalde M. Frecuencia de aislamientos microbiológicos y perfil de resistencia bacteriana en 13 clínicas y hospitales de alta complejidad en Santiago de Cali-Colombia. Infectio. 2014;18(1):3-11.

11. Levy SB, Marshall B. Antibacterial resistance worldwide: causes, challenges and responses. Nature medicine. 2004;10:S122-S9.

12. Boucher HW, Talbot GH, Bradley JS, Edwards JE, Gilbert D, Rice LB, et al. Bad Bugs, No Drugs: No ESKAPE! An Update from the Infectious Diseases Society of America. Clinical Infectious Diseases. 2009;48(1):1-12.

13. Cortes JA, Cuervo SI, Urdaneta AM, Potdevin G, Arroyo $P$, Bermudez $D$, et al. Identifying and controlling a multiresistant Pseudomonas aeruginosa outbreak in a Latin-American cancer centre and its associated risk factors. The Brazilian journal of infectious diseases : an official publication of the Brazilian Society of Infectious Diseases. 2009;13(2):99-103.
14. Berrouane YF, McNutt LA, Buschelman BJ, Rhomberg PR, Sanford MD, Hollis RJ, et al. Outbreak of severe Pseudomonas aeruginosa infections caused by a contaminated drain in a whirlpool bathtub. Clinical infectious diseases : an official publication of the Infectious Diseases Society of America. 2000;31(6):1331-7.

15. Breathnach AS, Cubbon MD, Karunaharan RN, Pope CF, Planche TD. Multidrug-resistant Pseudomonas aeruginosa outbreaks in two hospitals: association with contaminated hospital waste-water systems. J Hosp Infect. 2012;82(1):19-24.

16. Aoun M, Van der Auwera P, Devleeshouwer C, Daneau $D$, Seraj N, Meunier F, et al. Bacteraemia caused by nonaeruginosa Pseudomonas species in a cancer centre. $J$ Hosp Infect. 1992;22(4):307-16.

17. Mandell G, Dolin R, Bennett J, Mandell G, Bennett J. Mandell, Douglas, and Bennett's principles and practice of infectious diseases: Elsevier; 2009.

18. Peleg AY, Hooper DC. Hospital-Acquired Infections Due to Gram-Negative Bacteria. New England Journal of Medicine. 2010;362(19):1804-13.

19. Griffith SJ, Nathan C, Selander RK, Chamberlin W, Gordon S, Kabins S, et al. The epidemiology of Pseudomonas aeruginosa in oncology patients in a general hospital. Journal of Infectious Diseases. 1989;160(6):1030-6.

20. Kolar M, Sauer P, Faber E, Kohoutova J, Stosová T, Sedlackova $M$, et al. Prevalence and spread of Pseudomonas aeruginosa and Klebsiella pneumoniae strains in patients with hematological malignancies. The new microbiologica. 2009;32(1):67.

21. Rolston KV, Tarrand JJ. Pseudomonas aeruginosa-still a frequent pathogen in patients with cancer: 11-year experience at a comprehensive cancer center. Clinical infectious diseases. 1999;29(2):463-4.

22. Chatzinikolaou I, Abi-Said D, Bodey GP, Rolston KV, Tarrand JJ, Samonis G. Recent experience with Pseudomonas aeruginosa bacteremia in patients with cancer: retrospective analysis of 245 episodes. Archives of Internal Medicine. 2000;160(4):501-9.

23. Maschmeyer G, Braveny I. Review of the incidence and prognosis of Pseudomonas aeruginosa infections in cancer patients in the 1990s. European Journal of Clinical Microbiology \& Infectious Diseases. 2000;19 (12): 915-25

24. Cuervo SI, Cortes J, Bermúdez D, Martínez T, Quevedo $\mathrm{R}$, Arroyo CP. Infecciones intrahospitalarias en el instituto nacional de cancerología, Colombia, 20012002. Rev Colomb Cancerol. 2003;7(3):32-43.

25. Freifeld AG, Bow EJ, Sepkowitz KA, Boeckh MJ, Ito JI, Mullen CA, et al. Clinical practice guideline for the use of antimicrobial agents in neutropenic patients with cancer: 2010 update by the Infectious Diseases Society of America. Clinical Infectious Diseases. 2011;52(4):e56e93.

26. Network NCC. NCCN Clinical Practice Guidelines in Oncology: Prevention and treatment of cancer-related infections. V. 2.2009. Available online at www nccn org/ professionals/ physician_gls/ PDF/ infections pdf (Accessed 16 Decemeber 2010). 2009.

27. Keng MK, Sekeres MA. Febrile neutropenia in hematologic malignancies. Current hematologic malignancy reports. 2013;8(4):370-8. 
28. Talcott JA, Finberg R, Mayer RJ, Goldman L. The medical course of cancer patients with fever and neutropenia: clinical identification of a low-risk subgroup at presentation. Archives of internal medicine. 1988;148(12):2561-8

29. Innes H, Lim SL, Hall A, Chan SY, Bhalla N, Marshall E. Management of febrile neutropenia in solid tumours and lymphomas using the Multinational Association for Supportive Care in Cancer (MASCC) risk index: feasibility and safety in routine clinical practice. Supportive Care in Cancer. 2008;16(5):485-91.

30. Nguyen TC, Carcillo JA. Bench-to-bedside review: thrombocytopenia-associated multiple organ failure-a newly appreciated syndrome in the critically ill. Critical care. 2006;10(6):235.

31. Zhang Q, Smith JC, Zhu Q, Guo Z, MacDonald NE. A five-year review of Pseudomonas aeruginosa bacteremia in children hospitalized at a single center in southern China. International Journal of Infectious Diseases. 2012;16(8):e628-e32.
32. Horino T, Chiba A, Kawano S, Kato T, Sato F, Maruyama $Y$, et al. Clinical characteristics and risk factors for mortality in patients with bacteremia caused by Pseudomonas aeruginosa. Internal medicine. 2012;51(1):59-64.

33. Jeddi R, Ghédira H, Ben Amor R, Turki A, Kacem K, Ben Abdennebi Y, et al. Risk factors of septic shock in patients with hematologic malignancies and Pseudomonas infections. Hematology. 2011;16(3):160-5.

34. Zafer MM, Al-Agamy MH, El-Mahallawy HA, Amin MA, Ashour MSE-D. Antimicrobial resistance pattern and their beta-lactamase encoding genes among Pseudomonas aeruginosa strains isolated from cancer patients. BioMed research international. 2014;2014.

35. Tumbarello M, Repetto E, Trecarichi EM, Bernardini C, De Pascale G, Parisini A, et al. Multidrug-resistant Pseudomonas aeruginosa bloodstream infections: risk factors and mortality. Epidemiology and infection. 2011;139(11):1740-9. 